\title{
To Jean,
}

wife and closest companion for over fifty years, who knew all the bad times and supported us with good cheer, though her own wish for a peaceful and settled life was never realized. 
THIS PAGE INTENTIONALLY LEFT BLANK 